\title{
Noise Method of Electrical Resistance Measuring for Testing of Motion Control Systems
}

\author{
M. P. Vasylenko \\ Aviation Computer Integrated Complexes department \\ National Aviation University \\ Kyiv, Ukraine \\ vasylenkom.89@gmail.com
}

\begin{abstract}
Designed the method of measuring the electrical resistance of conductive material using its own thermal noise without the transmittance of electrical current through it that excludes the materials heating and other negative effects.
\end{abstract}

\section{Keywords- own thermal noise; electrical resistance; electromagnetic radiation}

\section{INTRODUCTION}

Nowadays motion control systems becomes more functional with more performance. But these advances causes such systems to become more and more sophisticated with lots of sensors. It is necessary to test and calibrate all the used instrumentation in order to obtain safe and reliable operation of the system. So it is necessary to improve the testing equipment to make such evaluation fester and easier or even to automate it.

Electrical resistance is inherent in all materials that are used by people. In many technical processes the resistance of the sensors provides valuable information about process parameters and allows to correct the process if necessary to achieve the needed result. That's the reason why the methods of measuring resistance continues to evolve.

Noise method of electrical resistance measuring allows to perform the non-destructive measuring of controlled objects resistance without the necessity of electrical current transmittance through it that allows to avoid oxidation or electrolytic dissociation.

\section{PROBLEM STATEMENT}

Such non-destructive measuring can be performed by the thermal noise measuring devices based on the dependency between the internal thermal noise voltage and the resistance of controlled object [1] - [3].

The thermal noise voltage equally depends on the resistance and temperature of the controlled object which causes the uncertainty in measurement results. Measurement error is also caused by the own noises of electronic components [3].

Measurement device [4] allows to reduce the influence of components own noise, but the influence of the temperature remains the same.
Known measurement device [5] allows to reduce such influences by using the exemplary resistor and calculating the ration between the resistances of exemplary resistor and controlled object. But often it is impossible to place the exemplary resistor near the controlled object and over time its resistance changes unpredictably especially at the high temperatures.

According to this it is necessary to improve the noise method of electrical resistance measuring.

\section{Method Description}

All the disadvantages, described above, are solved by the proposed thermal noise device for measuring electrical resistance [6].

The scheme of this device is given in Fig. 1.

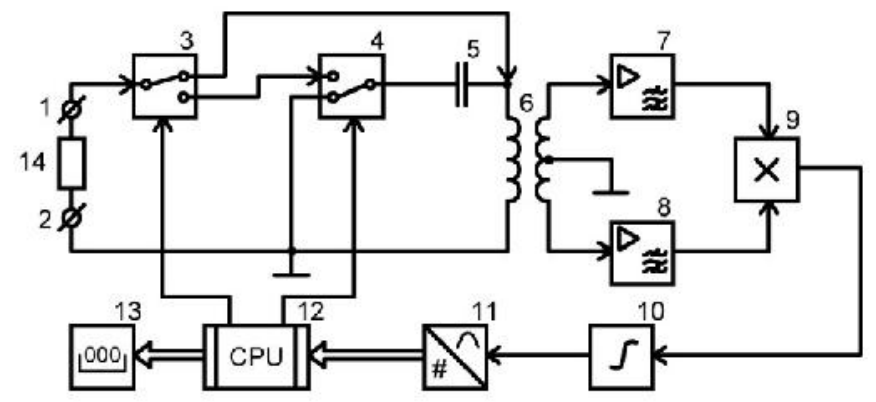

Fig. 1. Device schematic for measuring the electrical resistance: 1,2 are terminals; 3,4 are automatic switches; 5 is capacitor; 6 is mutual inductance coil; 7, 8 are amplifiers; 9 is multiplier; 10 is integrator; 11 is $\mathrm{ADC} ; 12$ is CPU; 13 is digital indicator; 14 is controlled object.

Controlled object 14 is connected to the measurement device by the terminals 1 and 2 . According to the program in CPU 12 automatic switch is switched into the upper position and automatic switch 4 is switched into the lower position because of the opposite polarity of control signal. Capacitor 5 and primary winding of mutual inductance coil 6 forms the parallel oscillatory circuit. Resonance frequency of oscillatory circuit can be found from formula:

$$
f_{0}=\frac{1}{2 \pi \sqrt{L C}}
$$


where $C$ is capacitance of capacitor $5 ; L$ is inductance of primary winding of mutual inductance coil 6 .

At the resonance frequency $f_{0}$ the oscillatory circuit has the active resistance, described by formula:

$$
R_{k}=\frac{L}{C r}
$$

where $r$ is resistance of primary winding.

At the high values of $Q$ factor of winding 6 resistance $r$ is small and resistance of oscillatory circuit $R_{k}$ is big:

$$
R_{k} \gg R_{x}
$$

where $R_{x}$ is resistance of controlled object 14 .

In this case the thermal noise generated by the controlled object 14 is not shunting by the external resistance of oscillatory circuit (no-load mode). Mean square voltage of oscillatory circuit can be found from Nyquist formula:

$$
\bar{U}_{n}=\sqrt{4 k T \Delta f R}
$$

where $k$ is Boltzmann constant; $T$ is temperature; $f$ is bandwidths of thermal noise.

Noise voltage (4) transforms into the secondary winding of mutual inductance coil 6 and gets amplified by the low bandwidth amplifiers 7 and 8, set to the resonance frequency (1) of oscillatory circuit. At the output of the multiplier 9 forms the voltage proportional to the square of noise voltage (4) and the product of own noise voltages of amplifiers 7 and 8 . These voltages are averaged by the integrator 10 .

Averaging reduces the product of amplifiers own voltages to zero. The DC voltage at the output of the integrator can be described by the formula:

$$
U_{1}=k_{1} k_{2} S \bar{U}_{n 1}^{2}
$$

where $k_{1}, k_{2}$ are amplification factors of amplifiers 7 and $8 ; S$ is transformation slope; $\bar{U}_{n 1}^{2}$ is variance of controlled objects 14 thermal noise. This voltage is converted by the ADC 11 into the digital code:

$$
N_{1}=\frac{4}{q} k_{1} k_{2} S k T \Delta f R_{x}
$$

where $q$ is the least digit of code.

Digital code (6) is stored into the memory block of the CPU 12.

Next, according to the program, the CPU changes the polarity of control signals and automatic switches 3 and 4 are switched into the opposite positions. It changes the configuration of oscillatory circuit to the serial. As shown in Fig. 2.
Resonance frequency (1) remains the same, so the following condition is fulfilled:

$$
2 \pi f_{0} L=\frac{1}{2 \pi f_{0} C}
$$

According to this, the current that flows through the primary winding of mutual inductance coil 6 is defined only by the resistance of controlled object 14 (short circuit mode). The mean square value of this current can also be obtained from Nyquist formula:

$$
\bar{I}_{n}=\sqrt{\frac{4 k T \Delta f}{R_{x}} .}
$$

Noise current (8) flows through the primary winding of mutual inductance coil 6 and creates the voltage on the secondary winding, that can be found from the formula:

$$
U_{n 2}=2 \pi f_{0} M \bar{I}_{n},
$$

where $M$ is coefficient of mutual inductance of coil 6 .

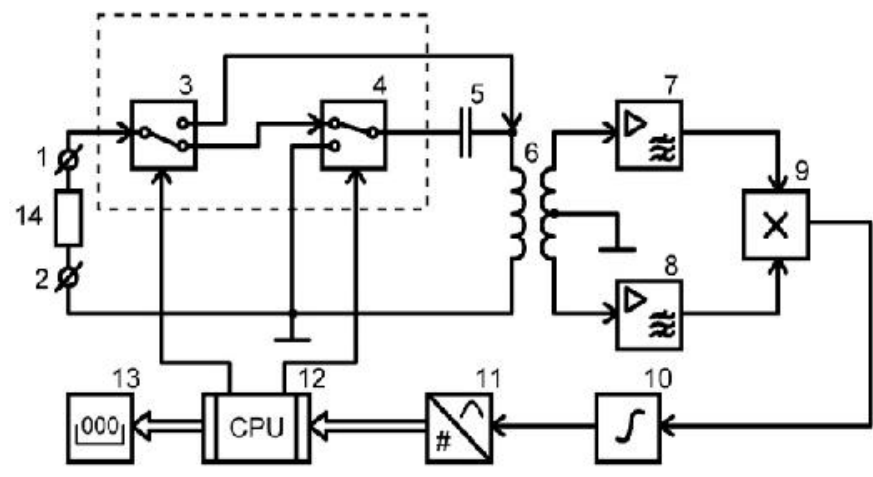

Fig. 2. Reconfiguration of the oscillatory circuit.

Noise voltage (9) is amplified by the amplifiers 7 and 8 . The output voltages of amplifiers are multiplied by the multiplier 9 and the output voltage is averaged by the integrator 10. The output voltage of the integrator can be described by formula

$$
U_{2}=k_{1} k_{2} S \bar{U}_{n 2}^{2}
$$

This voltage is also converted by the ADC into the digital code

$$
N_{2}=\frac{4}{q} k_{1} k_{2} S k \frac{T \Delta f}{R_{x}}\left(2 \pi f_{0} M\right)^{2},
$$

which is also stored into the memory block of the CPU.

Then the CPU performs the digital processing the first step if which is the digital code, that is the ratio of stored codes: 


$$
N_{3}=\frac{N_{1}}{N_{2}}=\left(\frac{R_{x}}{2 \pi f_{0} M}\right)^{2}
$$

The next step of processing is the square root from code (12):

$$
N_{2}=\sqrt{N_{3}}=\frac{R_{x}}{2 \pi f_{0} M},
$$

and the multiplying of this code (12) on the constant coefficient:

$$
N_{5}=N_{4}\left(2 \pi f_{0} M\right)=R_{x}
$$

Resulting code (14) is transformed into the form suitable for the user and shown on the digital indicator 13 .

Developed measuring device provides the direct measuring of electrical resistance independently from its temperature. Reducing of amplifiers own noise influence allows to measure electrical resistance of the controlled object in the wide range. The influence of amplification coefficients instability $\left(k_{1}\right.$ and $k_{2}$ ), instability of transformation slope $(S)$, instability of bandwidth $(f)$ and the error of the ADC limited bits $(q)$ allows to increase accuracy of resistance measuring in comparison with similar equipment.

In order to eliminate the non-informative low frequency noises it is necessary to pick the thermal noise in the range from 150 to $400 \mathrm{kHz}$ with 5-10 kHz bandwidth.

Changing of oscillatory circuit configuration can be reliably and easily performed by the magnetically controlled relays, connected to the logical outputs of the CPU.

The measurement process duration is determined by the time constant of the integrator that is picked big enough according to the statistic nature of the thermal noise.

\section{EXPERIMENTAL RESULTS}

Method accuracy was checked by measuring the reference resistances. Results are shown in Table 1.

Provided data shows, that proposed method allows to measure electrical resistance with $0.04 \%$ average error.

\section{CONCLUSIONS}

Proposed measuring device allows to measure the electrical resistance without the transmittance of the electrical current through the controlled object that allows to avoid the negative effects, such as local heating of the object, its oxidation or electrolytic dissociation. It also allows to perform measurement in the environments with flaming or explosion hazard.

Device is suitable for testing of motion control systems equipment. Use of microprocessor as the heart of the device allows to automate the testing process in the future and use this device as the self-testing equipment.
Measuring device can also be used as the part of on-board testing equipment to test the resistive sensors of different

\begin{tabular}{|c|c|c|c|}
\hline \multirow{2}{*}{ Num. } & \multicolumn{2}{|c|}{ Electrical Resistance, Ohm } & \multirow{2}{*}{ Errors, \% } \\
\hline & Reference & Noise M ethod & \\
\hline 1 & 100 & 100.25 & 0.25 \\
\hline 2 & 200 & 200.30 & 0.15 \\
\hline 3 & 300 & 299.80 & 0.07 \\
\hline 4 & 400 & 400.33 & 0.08 \\
\hline 5 & 500 & 499.70 & 0.06 \\
\hline 6 & 600 & 599.90 & 0.02 \\
\hline 7 & 700 & 700.25 & 0.04 \\
\hline 8 & 800 & 800.17 & 0.02 \\
\hline 9 & 900 & 900.19 & 0.02 \\
\hline 10 & 1000 & 999.83 & 0.02 \\
\hline 11 & 1100 & 1099.89 & 0.01 \\
\hline 12 & 1200 & 1200.20 & 0.02 \\
\hline 13 & 1300 & 1300.31 & 0.02 \\
\hline 14 & 1400 & 1399.77 & 0.02 \\
\hline 15 & 1500 & 1500.15 & 0.01 \\
\hline 16 & 1600 & 1600.23 & 0.01 \\
\hline 17 & 1700 & 1699.85 & 0.01 \\
\hline 18 & 1800 & 1799.88 & 0.01 \\
\hline 19 & 1900 & 1900.14 & 0.01 \\
\hline 20 & 2000 & 2000.22 & 0.01 \\
\hline & & Average Error & 0.04 \\
\hline
\end{tabular}
aircraft systems.

TABLE I. EXPERIMENTAL RESULTS

\section{REFERENCES}

[1] . V Savateev, Noise Thermometry. Leningrad, Energoatomizdat, 1987. (in Russian)

[2] Aldert Van der Ziel, Noise in measurements. John Wiley \& Sons Inc, 1976.

[3] Aldert Van der Ziel, Noise. Sources, Characterization, Measurement Prentice Hall, 1971.

[4] Patent of Ukraine №14264, IPC G01R27/00, Thermal noise method of measuring high resistances, Skrypnyk Yu. O., Kurko V. R., and Kalameyets T. P., 2006. (in Ukrainian)

[5] Patent of Ukraine №62760, IPC G01N27/02, Condutometric measurer of moisture, Yu. O. Skrypnyk, and V. R. Kurko, 2003 (in Ukrainian)

[6] Patent of Ukraine №74499, Thermal noise measurer of electrical resistance, Yu. O. Skrypnyk, O. A. Lisovkkyi, and M. P. Vasylenko, 2012. (in Ukrainian) 